BMJ Open

Diabetes

Research

\& Care

\title{
Diabetes hinders community-acquired pneumonia outcomes in hospitalized patients
}

\author{
M Martins, ${ }^{1,2,3} \mathrm{~J} \mathrm{M} \mathrm{Boavida,}{ }^{1} \mathrm{~J}$ F Raposo, ${ }^{1,2}$ F Froes, ${ }^{4}$ B Nunes, ${ }^{5}$ R T Ribeiro,,${ }^{1,2}$ \\ M P Macedo, ${ }^{1,2}$ C Penha-Gonçalves ${ }^{3}$
}

To cite: Martins M, Boavida JM, Raposo JF, et al. Diabetes hinders communityacquired pneumonia outcomes in hospitalized patients. BMJ Open Diabetes Research and Care 2016;4: e000181. doi:10.1136/ bmjdrc-2015-000181

Received 10 December 2015 Revised 4 April 2016 Accepted 28 April 2016
CrossMark

For numbered affiliations see end of article.

Correspondence to Dr Carlos Penha-Gonçalves; cpenha@igc.gulbenkian.pt

\section{ABSTRACT}

Objectives: This study aimed to estimate the prevalence of diabetes mellitus (DM) in hospitalized patients with community-acquired pneumonia (CAP) and its impact on hospital length of stay and inhospital mortality.

Research design and methods: We carried out a retrospective, nationwide register analysis of CAP in adult patients admitted to Portuguese hospitals between 2009 and 2012. Anonymous data from 157291 adult patients with CAP were extracted from the National Hospital Discharge Database and we performed a DM-conditioned analysis stratified by age, sex and year of hospitalization.

Results: The 74175 CAP episodes that matched the inclusion criteria showed a high burden of DM that tended to increase over time, from $23.7 \%$ in 2009 to $28.1 \%$ in 2012. Interestingly, patients with CAP had high DM prevalence in the context of the national DM prevalence. Episodes of CAP in patients with DM had on average 0.8 days longer hospital stay as compared to patients without DM $(p<0.0001)$, totaling a surplus of 15370 days of stay attributable to DM in 19212 admissions. In-hospital mortality was also significantly higher in patients with CAP who have DM (15.2\%) versus those who have DM (13.5\%) ( $p=0.002)$.

Conclusions: Our analysis revealed that DM prevalence was significantly increased within CAP hospital admissions, reinforcing other studies' findings that suggest that DM is a risk factor for CAP. Since patients with CAP who have DM have longer hospitalization time and higher mortality rates, these results hold informative value for patient guidance and healthcare strategies.

\section{INTRODUCTION}

Diabetes mellitus (DM) is a significant public health burden, representing one of the most common chronic diseases worldwide and being associated with high morbidity and mortality. ${ }^{1}$ The increasing prevalence of DM and other comorbidities has been suggested as a driving factor for the rising burden of infection-related hospitalizations. ${ }^{2-4}$ Respiratory infections are among the major infections associated with diabetes. ${ }^{5}$ Community-acquired

\section{Key messages}

- Diabetes mellitus increases the risk for hospitalisation of patients with community-acquired pneumonia.

- Episodes of community-acquired pneumonia in patients with diabetes mellitus require a longer hospital stay.

- Diabetes mellitus impacts on mortality of hospitalized patients with community-acquired pneumonia.

pneumonia (CAP) is one of the most frequent infections requiring hospital admissions in developed countries; ${ }^{6}$ it ranks among the top causes of death and is a major driver of healthcare utilization and cost. $^{7-9}$ In Portugal, a retrospective study with data from 2000 to 2009 reported that admissions for CAP represented $3.7 \%$ of total admissions of adult patients. ${ }^{10}$ Association of DM with CAP hospitalizations has been suggested previously. ${ }^{8} 9^{11-14}$ However, information regarding the burden and outcomes of DM in patients with CAP, and particularly its relationship with hospitalization and in-hospital mortality, is still limited.

Recommendations of flu and pneumococcal vaccination of patients with $\mathrm{DM}$ by medical societies and several health authorities reflect the notion that people with diabetes are at increased risk of respiratory infections. ${ }^{1}$ Within the European context, Portugal presents one of the highest rates of DM. The PREVADIAB study estimated that the nationwide prevalence of DM in 2009 was $11.7 \%$ within the adult population (2079 years). ${ }^{15}$ Since 2003 , the annual rate of new DM cases in Portugal increased by $3.8 \%$ on average. ${ }^{16}$ In 2012, people with diabetes represented $14 \%$ of the patients admitted to the Portuguese hospital public system, and $23.5 \%$ of in-hospital mortality involved patients with DM. ${ }^{16}$

Robust information on the impact of DM among the population hospitalized with CAP 
is critical to ascertain the differential risk of patients with DM and to design effective preventive care strategies. Hence, we have carried out a retrospective analysis of the Portuguese hospital registers of adult patients hospitalized with CAP between 2009 and 2012. We aimed to estimate the prevalence of DM among this population and the impact of DM on length of hospital stay and in-hospital mortality.

\section{METHODS}

\section{Data sources}

Data were retrieved from the Central Administration of the Health System of the Portuguese Ministry of Health that contains administrative and clinical data on all admissions to National Health System hospitals, covering the vast majority of Portugal's mainland population.

The clinical information, including case identification (namely the clinical diagnosis of CAP and DM) and procedures, is encoded from the details of the hospital discharge report by medical doctors who were specially trained in hospital coding, using the International Classification of Diseases, 9th Revision Clinical Modification (ICD-9-CM). ${ }^{17}$ The coding is audited periodically by the ACSS (Administração Central do Sistema de Saúde; Central Administration of the Health System) in Portugal. This type of methodology has been previously used and validated in other studies. ${ }^{10}$ Anonymized data were extracted from the National Hospital Discharge Database from 2009 to 2012. Research was conducted according to the principles of the Declaration of Helsinki. Anonymized data were retrieved from an administrative database. Once it is not possible to identify, by any means, an individual patient, ethical approval was not required and informed consent not sought.

\section{Study population and eligibility}

Subjects included in the study were patients aged 2079 years with CAP diagnosis as the main cause of admission (ICD-9-CM 480-486 and 487.0). Data for analysis were clean from day cases and hospitalizations $>90$ days. To avoid overestimation from repeated admissions, diagnostic codes recorded within 28 days of one another for the same individual were attributed to a single infection episode, with the index date defined by the first diagnostic code. We excluded from the analysis individuals who were immunocompromised by anticancer or immunosuppressive treatment (external cause of disease code E933.1) and transplant recipients (V42) and patients with HIV (ICD-9-CM 042-044). Within the selected subjects, we looked for concomitant diagnosis of diabetes in the discharge records (ICD-9-CM 250), to differentiate two groups of individuals, CAP with or without DM. Nationwide DM statistics from 2009 to 2012 were retrieved from the national diabetes prevalence study (PREVADIAB). ${ }^{15}$ For comparison purposes, age range (20-79 years) and age stratification (age groups: 20-39,
40-59 and 60-79 years) followed the PREVADIAB study. Information on the population size of mainland Portugal was provided by the Instituto Nacional de Estatística (http://www.ine.pt) and corresponds to estimates of the resident population at the end of each year, according to age and sex.

\section{Data analysis}

CAP with DM (CAP-DM) was compared with CAP without DM for age and gender biases, hospitalization time and mortality rate, across age groups and over the 2009-2012 period. Categorical variables were described using counts and percentages while continuous variables were expressed as the mean and SD. The median and IQRs are also shown to take into account the nonnormal distribution of continuous variables under analysis (Shapiro-Wilk test). Significant differences between groups were detected by $\chi^{2}$ test or Fisher exact test for categorical variables, and by the Student's t-test or Mann-Whitney test for continuous variables, as appropriate. Analysis of hospital length of stay and in-hospital mortality used univariate analysis of variance (ANCOVA), adjusted for age and gender. The Cochran-Armitage test was performed for a trend analysis of diabetes prevalence changes over time (from 2009 to 2012). All the calculations presented were obtained using the statistical software package PASW Statistics 19.0 (SPSS Inc.) and the Microsoft Excel 2007 spreadsheet (Microsoft Corp., Redmond, Washington, USA). A p value of $<0.05$ was considered statistically significant. All reported $p$ values are two-tailed.

\section{RESULTS}

From the initial 157291 registers of CAP admissions, from 2009 to 2012 (37 989-40 200/year), after applying the exclusion criteria and selecting the target age range (20-79), we analyzed 74175 episodes of CAP hospitalizations, of which 19212 (25.9\%) corresponded to people with DM (table 1).

The median age of the cohort was 72 (IQR, 6576 years) for CAP-DM, and 67 (IQR, 53-75 years) for CAP without DM, indicating that patients with DM were significantly older $(\mathrm{p}<0.0001)$. We performed an age-stratified analysis of 2009-2012 data to ascertain whether the observed effects are more prominent in specific strata. In the three age groups (20-39; 40-59 and 60-79 years), patients with CAP-DM were always significantly older $(\mathrm{p}<0.0001)$ (table 2$)$. Although the age distribution was skewed to old ages both in patients with CAP with or without DM, it is worth noting that the age group 60-79 represented over $86 \%$ of the patients with CAP-DM, while the age group 20-39 was underrepresented $(1.2 \%)$ (table 2 and figure 1$)$. The median age showed a trend to increase from 2009 to 2012, most prominently in patients with CAP without DM (from 66 years in 2009 to 69 years in 2012), but the patients 
Table 1 Patients with CAP with and without DM hospitalized in Portugal between 2009 and 2012 (age range 20-79 years)

\begin{tabular}{|c|c|c|c|}
\hline & CAP-DM & CAP-without-DM & p Value \\
\hline n (\%) & $19212(25.9)$ & $54963(74.1)$ & \\
\hline Mean $\pm S D$ Age, years & $69.4 \pm 9.2$ & $62.7 \pm 14.9$ & \\
\hline Median (IQR) Age, years & $72(65-76)$ & $67(53-75)$ & $<0.0001$ \\
\hline Male/female, n (\%) & $11231 / 7981$ (58.5/41.5) & 34 027/20 $936(61.9 / 38.1)$ & $<0.0001$ \\
\hline Mean $\pm S D$, hospitalization days & $12.0 \pm 10.5$ & $11.2 \pm 10.1$ & \\
\hline Median (IQR) hospitalization days & $9(6-14)$ & $8(5-13)$ & $<0.0001$ \\
\hline In-hospital mortality, n (\%) & $2918(15.2)$ & $7427(13.5)$ & 0.002 \\
\hline \multicolumn{4}{|c|}{$\begin{array}{l}\text { Significant differences between groups were detected by means of } \chi^{2} \text { test for categorical variables, and by the Student's t-test or } \\
\text { Mann-Whitney test for continuous variables, as appropriate. Univariate analysis of variance (ANCOVA), adjusted for age and gender was } \\
\text { used to compare hospital length of stay and in-hospital mortality between the two groups. } \\
\text { Significant } p \text { values are highlighted in bold. }\end{array}$} \\
\hline
\end{tabular}

with CAP-DM were always significantly older $(\mathrm{p}<0.0001)$ (table 3).

The prevalence of CAP, irrespective of DM co-occurrence, was higher among men than women (table 1). However, the male/female ratio was significantly different between the two groups $(\mathrm{p}<0.0001)$, with patients with CAP-DM showing a lower proportion of men $(58.5 \%)$ when compared to patients with CAP without DM (61.9\%) (table 1). This gender difference increased from the age group 20-39 years to 4059 years, then decreasing in the age group 60-79 years, possibly reflecting the decreased male/female ratio in the general elderly population (table 2). Nevertheless, men were more likely to experience CAP infections than women in all age groups. During the analysis period, the percentage of males varied between $57.5 \%$ and $59.5 \%$ in CAP-DM, and 60.6\%-63.0\% in CAP without DM (table 3). Interestingly, the burden of DM in CAP was more pronounced in women $(25.1 \%)$ than in men $(22.8 \%)$.

Analysis adjusted for sex and age revealed that the average length of stay was significantly longer in CAP-DM cases $(\mathrm{p}<0.0001)$, with an average length of stay of $12.0 \pm 10.5$ vs $11.2 \pm 10.1$ days. This difference was observed for all age groups but was highest in the younger age stratum (20-39 years; +2.5 days) in comparison with the older stratum (60-79 years; +0.2 days). In the 2009-2012 period, the average length of stay was consistently higher in CAP-DM cases when compared to CAP without DM, with the difference in length of stay varying from +0.8 to +1.0 days totalling an estimated increase of 15370 days of stay attributable to DM in over 19000 episodes (table 3 ), which represent $\sim 0.93 \%$ of total hospital admissions (according to Froes et $a l^{10}$ ).

Interestingly, in-hospital mortality (20-79 years), adjusted for sex and age, was significantly higher in patients with CAP who have DM (15.2\%) as compared to patients with CAP without DM (13.5\%) ( $\mathrm{p}=0.002)$ (table 1). From 2009 to 2012, in-hospital mortality was always higher in patients with CAP who have DM (14.8$16.0 \%)$ as compared to patients with CAP without DM (12.7-14.3\%) (table 3). However, when analyzing by age group, increased mortality of patients with DM was only observed in the youngest age group. Furthermore, in-hospital mortality in older strata revealed that patients with CAP without DM show a trend to higher mortality. This apparent discrepancy is probably due to the underrepresentation of the youngest age group (20-39 years) in patients with CAP-DM $(1.2 \%)$ as compared to patients with CAP without DM (10.2\%) (table 2$)$.

We found that the DM burden in CAP episodes steadily increased over time, from $23.7 \%$ in 2009 to $28.1 \%$ in 2012 (table 3), representing a trend of increasing DM prevalence among patients with CAP (Cochran-Armitage test; $\mathrm{p}$ value $\left.<2.2 \times 10^{-16}\right)$. This may result in part from the impact of population ageing since, in our study, there was an increase in the representation of individuals in the 60-79 years age group from $67.5 \%$ in 2009 to $75.4 \%$ in 2012 (data not shown).

\section{DISCUSSION}

Diabetes mellitus has been frequently associated with respiratory infections. We performed a large cohort retrospective study that allowed detailed nationwide estimates of DM prevalence in CAP hospital admissions as well as comparisons to national DM prevalence in Portugal. We found a high burden of DM in patients hospitalized with CAP $(25.9 \%)$, in agreement with the high rates obtained in previous studies of $21.4 \%$ and $16 \%$ performed elsewhere. ${ }^{12} 18$ The burden of DM increased over the period under analysis (2009-2012), similar to what was observed in a recent study estimating the burden of CAP, between 1997 and 2011, in the $\mathrm{UK}^{19}$ In line with studies that suggest that patients with $\mathrm{DM}$ are at higher risk for CAP (reviewed in $^{8}$ ), we observed that the DM prevalence in CAP admissions between 2009 and 2012 was consistently higher, and more than double, when compared to the estimations of the DM prevalence in Portugal (figure 2).

Our study covered admissions from all Portuguese public hospitals over a period of 4 years to analyze the impact of DM in CAP hospitalizations. We corrected our analyses for gender and age and excluded immunocompromised individuals. Nevertheless, these analyses have 


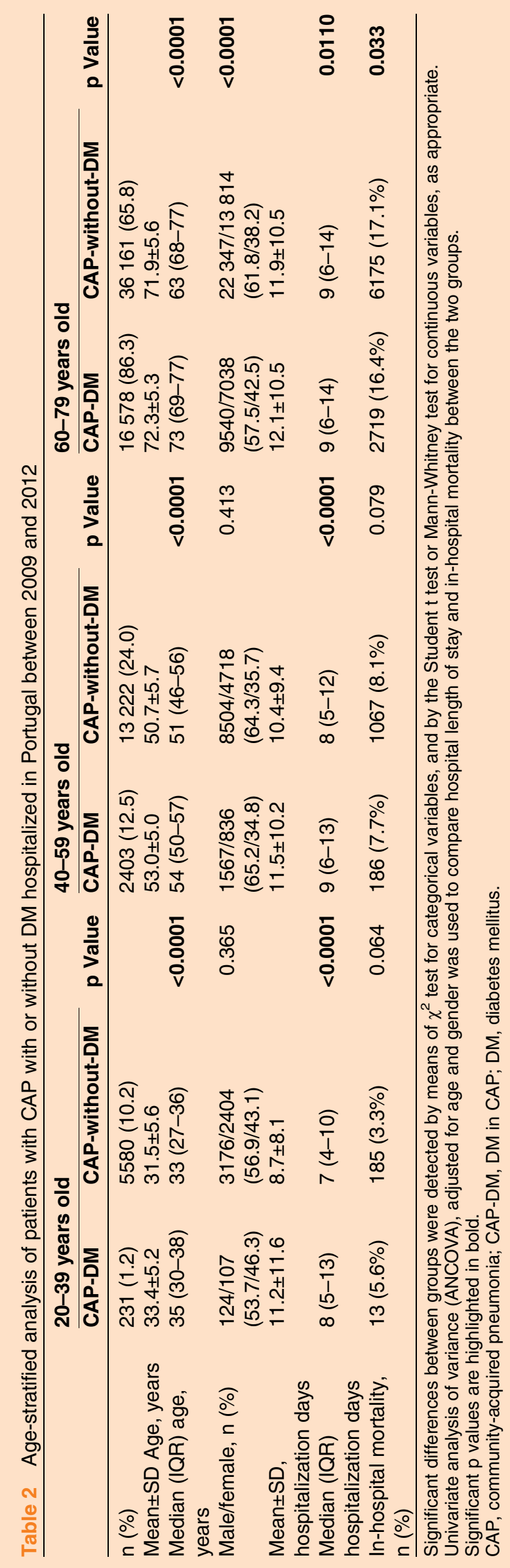

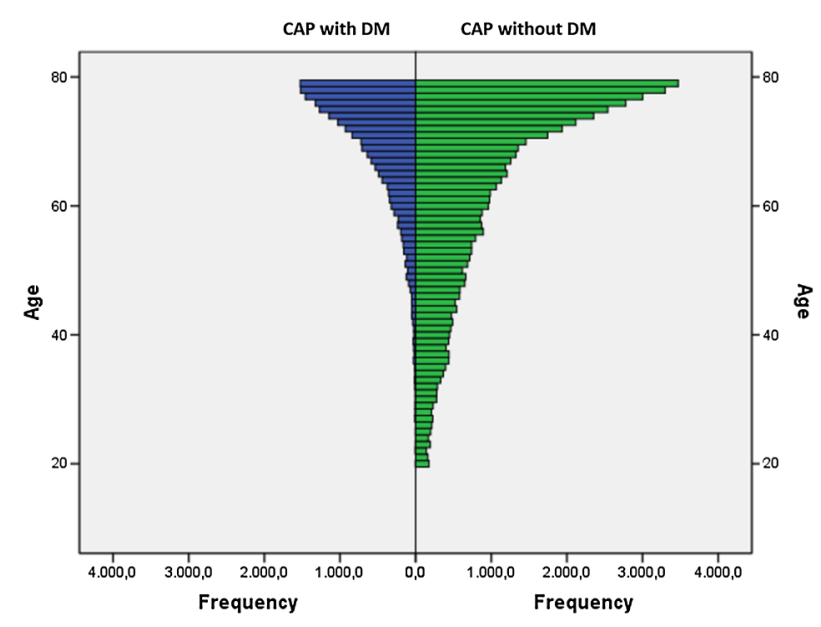

Figure 1 Age distribution of CAP with diabetes and CAP without diabetes (2009 to 2012) shows a stronger skewedness to old ages in patients with CAP-DM. CAP, community-acquired pneumonia; CAP-DM, DM in CAP; DM, diabetes mellitus.

several inherent limitations. The data are derived from a database that did not contain direct clinical measures and we did not have access to clinical records. Therefore, we could not include in the analyses possible confounding factors, namely related to diabetes and CAP severity, therapies and vaccination, smoking history or lifestyles. We have also to take into account that diabetes is a frequent cause for hospitalization, and that this potential bias could overestimate the incidence of diabetes in respiratory infections and lead one to suspect that diabetes is a risk factor for susceptibility. ${ }^{20}$ In our study, case identification was based in medical record information following the international coding system that was applied by specifically trained medical staff in according a methodology that was previously validated. ${ }^{10}$ Thus, we reason that misclassification in the hospital discharge database is likely to be relatively small, although underestimation of diabetes in hospital settings has been reported elsewhere. ${ }^{21}$ Although we did not follow a study design specific to compare the target population with the general population, ${ }^{22}$ we consider that contrasting our results with the general population in Portugal will help to contextualize the burden of DM in hospitalized patients with CAP.

In agreement with two prospective studies in Spain $^{12}{ }^{18}$ we found that age distribution in CAP-DM patients was skewed to older ages as compared to CAP patients without DM, which reflects the age of DM onset. The median age of CAP admissions showed a trend to increase from 2009 to 2012, in accordance with Froes et $a l,{ }^{10}$ which also reported a $5 \%$ increase in the average age of patients admitted with CAP between 2000 and 2009 in Portugal. As reported in other studies, CAP infections affected more men than women. ${ }^{10} 12 \quad 18$ Interestingly, the prevalence of diabetes among CAP admissions was higher in women than in men $(25.1 \%$ vs 


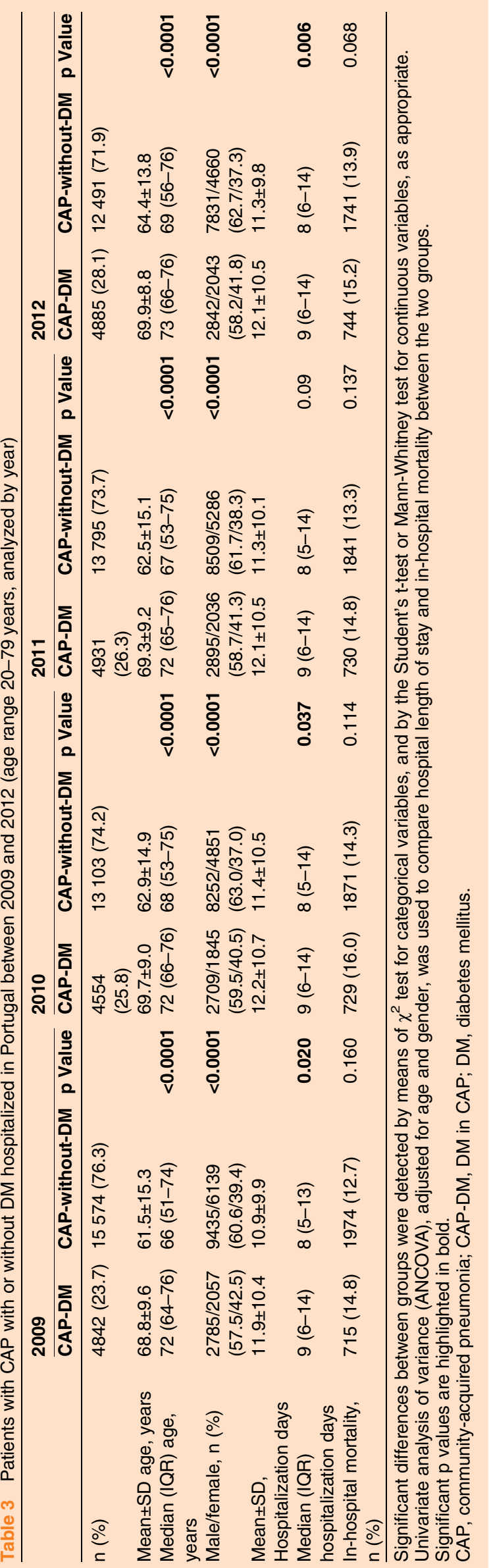

$22.8 \%$ ), as opposed to what is observed in the general population (10.2\% vs $14.6 \%$ ) (figure 3), suggesting a higher risk of women with DM to develop CAP infections.

We found a longer length of stay in patients with CAP with DM, in line with other studies indicating significantly higher rates of hospitalization in patients with CAP-DM. ${ }^{11}{ }^{12}$ This may be attributable to effects of diabetes mellitus in exacerbating underlying comorbidities, which is supported by the observation that in the young age range (20-39 years) patients with CAP-DM showed a higher length of stay (table 2). Indeed, Di Yacovo et $a l^{18}$ performed an observational analysis of a prospective cohort of immunocompetent hospitalized adults with CAP and found that patients with DM had distinctive clinical features. Patients with DM had more in-hospital acute metabolic complications ${ }^{18}$ and more severe pneumonia. ${ }^{12}{ }^{23}$ Additionally, it has been shown that patients with CAP with diabetes have a higher frequency of other concomitant conditions,${ }^{12}$ including chronic pulmonary diseases (eg, $32.6 \%$ in CAP with $\mathrm{DM}^{18}$ and $22 \%$ in pneumonia with $\mathrm{DM}^{23}$ ).

The impact of DM in hospital mortality (table 1) was mainly contributed by the 20-39 age group which showed higher mortality (table 2). This is in agreement with several other studies where patients with DM had a higher risk of death from $\mathrm{CAP}^{12}{ }^{24}$ or from pneumonia. ${ }^{25}$ Kornum et $a l^{11}$ also reported that people with diabetes < 40 years (15-39 years) were three times more likely to be hospitalized with pneumonia than individuals without diabetes of similar age, with the relative risk decreasing with ageing in individuals with DM. As expected, type 1 diabetes was over-represented in the youngest age group (20-39 years; $26.8 \%$ ) as compared to the $40-59$ and $60-79$ age groups $(3.3 \%$ and $1.4 \%$, respectively). Nevertheless, individuals with type 1 diabetes in the youngest age group showed lower length of hospital stay $(10.2 \pm 7.1$ vs $11.2 \pm 11.6)$ and lower in-hospital mortality $(4.2 \%$ vs $5.6 \%)$ than the total group with DM (type 1+type 2 diabetes), indicating that inclusion of patients with type 1 diabetes did not influence our analysis. On the other hand, we could not ascertain whether the severity of metabolic imbalances, namely ketoacidosis, would contribute to the observed increased mortality in young patients with diabetes.

Taken together, our nationwide results are in line with other reports, suggesting that patients with DM are at increased risk of $\mathrm{CAP}^{8}{ }^{9}{ }^{11-14}$ This longitudinal study provides indications that patients with DM acquiring CAP are older, have a longer hospitalization time and have higher mortality rates as compared to patients with CAP without DM. Our study also highlights that the relative impact of diabetes was greatest in younger adults (20-39 years) and in women. Thus, this nationwide study identified people with DM as a priority group for adoption of general measures to prevent CAP (eg, smoking cessation and control of chronic illnesses), and more specifically for flu and prophylactic pneumococcal 
Figure 2 Diabetes prevalence in CAP admissions and the general Portuguese population from 2009 to 2012. Diabetes prevalence in CAP admissions consistently progressed from 2009 to 2012 , as compared to the general Portuguese population, in the age range $20-79$ years. CAP, community-acquired pneumonia.
Portuguese population

CAP admissions

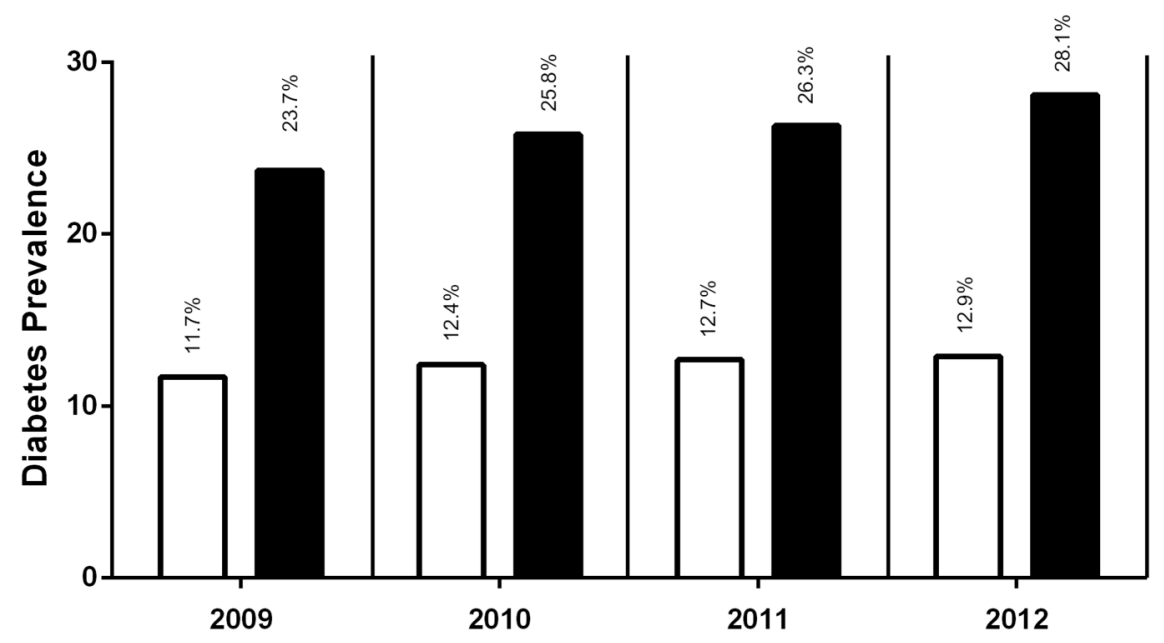

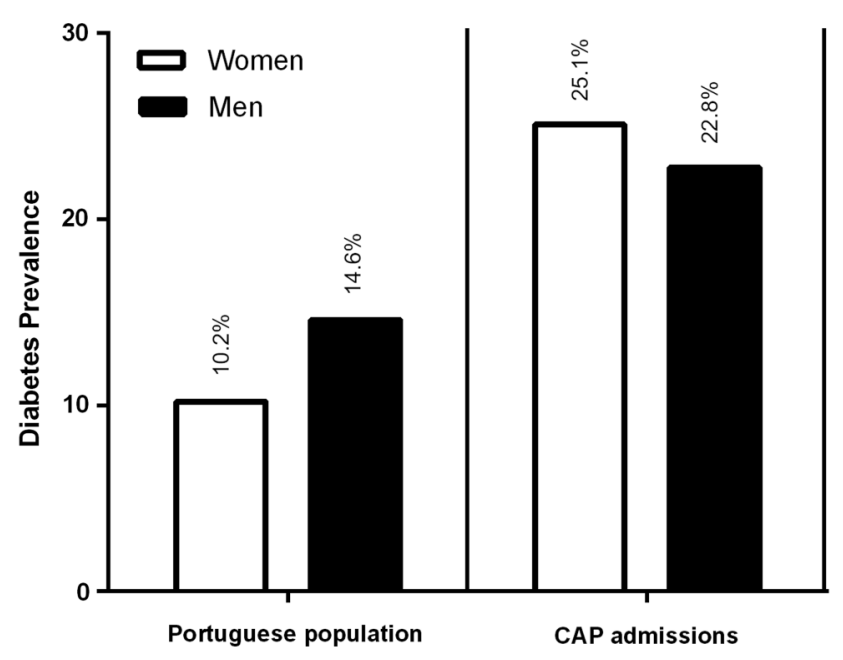

Figure 3 Gender differences in diabetes prevalence in 2009 in the general Portuguese population patients and in patients with CAP. Diabetes prevalence is higher in women as compare to men in CAP admissions but not in the general Portuguese population.

vaccination. These results have informative value for strategies of patient guidance and future healthcare policies, particularly in people with diabetes under 40 years and in women with DM.

\section{Author affiliations}

${ }^{1}$ APDP—Diabetes Portugal (Education and Research Centre/APDP-ERC), Lisboa, Portugal

${ }^{2}$ Chronic Diseases Research Center (CEDOC), NOVA Medical School-FCM, Universidade Nova de Lisboa, Lisboa, Portugal

${ }^{3}$ Instituto Gulbenkian de Ciência, Oeiras, Portugal

${ }^{4}$ Service of Pneumology, Hospital Pulido Valente, Centro Hospitalar Lisboa Norte, Lisboa, Portugal

${ }^{5}$ Department of Epidemiology, Instituto Nacional de Saúde Dr. Ricardo Jorge, Lisboa, Portugal

Acknowledgements The work presented in this paper was distinguished by the Sociedade Portuguesa de Diabetologia with the prize Professor Manuel Hargreaves/SPD/Novartis.
Contributors MM, JMB, JFR and MPM designed the study. MM and JMB collected the data. MM, FF, BN, RTR, MPM and CP-G provided quality control and analyzed the data. MM, FF, JMB and CPG interpreted the data. MM and $\mathrm{CP}-\mathrm{G}$ wrote the article. All authors contributed to the critical revision and final approval of the manuscript.

Funding This work was supported by a Pfizer Grant to Ernesto Roma Foundation, Grant number FER2014/01 to MM.

Competing interests None declared.

Provenance and peer review Not commissioned; externally peer reviewed.

Data sharing statement The raw data used in this study are held by the Central Administration of the Health System of the Portuguese Ministry of Health.

Open Access This is an Open Access article distributed in accordance with the Creative Commons Attribution Non Commercial (CC BY-NC 4.0) license, which permits others to distribute, remix, adapt, build upon this work noncommercially, and license their derivative works on different terms, provided the original work is properly cited and the use is non-commercial. See: http:// creativecommons.org/licenses/by-nc/4.0/

\section{REFERENCES}

1. American Diabetes Association. Standards of medical care in diabetes-2014. Diabetes Care 2014;37(Suppl 1):S14-80.

2. Bardsley M, Blunt I, Davies S, et al. Is secondary preventive care improving? Observational study of 10-year trends in emergency admissions for conditions amenable to ambulatory care. BMJ 2013;3:e002007.

3. Trotter CL, Stuart JM, George R, et al. Increasing hospital admissions for pneumonia, England. Emerging Infect Dis 2008;14:727-33.

4. Korbel L, Spencer JD. Diabetes mellitus and infection: an evaluation of hospital utilization and management costs in the United States. J Diabetes Complicat 2015;29:192-5.

5. Smith SA, Poland GA, American Diabetes Association. Influenza and pneumococcal immunization in diabetes. Diabetes Care 2004;27(Suppl 1):S111-13.

6. Mandell LA, Wunderink RG, Anzueto A, et al. Infectious Disease Society of America/American Thoracic Society consensus guidelines on the management of community-acquired pneumonia in adults. Clin Infect Dis 2007;44(Suppl 2):S27-72.

7. Polsky D, Bonafede M, Suaya JA. Comorbidities as a driver of the excess costs of community-acquired pneumonia in U.S. commercially-insured working age adults. BMC Health Serv Res 2012;12:379.

8. Torres A, Blasi F, Dartois N, et al. Which individuals are at increased risk of pneumococcal disease and why? Impact of COPD, asthma, smoking, diabetes, and/or chronic heart disease on 
community-acquired pneumonia and invasive pneumococcal disease. Thorax 2015;70:984-9.

9. Millett ERC, De Stavola BL, Quint JK, et al. Risk factors for hospital admission in the 28 days following a community-acquired pneumonia diagnosis in older adults, and their contribution to increasing hospitalisation rates over time: a cohort study. BMJ Open 2015:5:e008737.

10. Froes F, Diniz A, Mesquita M, et al. Hospital admissions of adults with community-acquired pneumonia in Portugal between 2000 and 2009. Eur Respir J 2013;41:1141-6.

11. Kornum J, Thomsen R, Riis A, et al. Diabetes, glycemic control, and risk of hospitalization with pneumonia. Diabetes Care 2008;8:1541-5.

12. Falguera M, Pifarre R, Martin A, et al. Etiology and outcome of community-acquired pneumonia in patients with diabetes mellitus. Chest 2005;128:3233-9.

13. Almirall J, Bolíbar I, Serra-Prat M, et al., Community-Acquired Pneumonia in Catalan Countries (PACAP) Study Group. New evidence of risk factors for community-acquired pneumonia: a population-based study. Eur Respir J 2008;31:1274-84.

14. Ishida $\mathrm{T}$, Tachibana $\mathrm{H}$, Ito $\mathrm{A}$, et al. Clinical characteristics of severe community-acquired pneumonia among younger patients: an analysis of 18 years at a community hospital. J Infect Chemother 2014;20:471-6.

15. Gardete-Correia L, Boavida JM, Raposo JF, et al. First diabetes prevalence study in Portugal: PREVADIAB study. Diabet Med 2010;27:879-81.

16. Sociedade Portuguesa de Diabetologia. Diabetes: Factos e Números 2013-Relatório Anual do Observatório Nacional da Diabetes 2013. http://www.apdp.pt/index.php/diabetes/ factos-e-numeros/diabetes-factos-e-numeros-2013. Date last updated: 24 February2015 (accessed 22 Sep 2014).

17. International Classification of Diseases, 9th Revision-Clinical Modification. http://www.cdc.gov/nchs/icd/icd 9.htm. Date last updated: 18 June 2013 (accessed 27 Jun 2014).

18. Di Yacovo S, Garcia-Vidal C, Viasus D, et al. Clinical features, etiology, and outcomes of community-acquired pneumonia in patients with diabetes mellitus. Medicine (Baltimore) 2013;92: $42-50$.

19. McDonald $\mathrm{HI}$, Nitsch D, Millett ER, et al. New estimates of the burden of acute community-acquired infections among older people with diabetes mellitus: a retrospective cohort study using linked electronic health records. Diabet Med 2014;31:606-14.

20. Smith SA, Poland GA. The use of influenza and pneumococcal vaccines in people with diabetes. Diabetes Care 2000;23:95-108.

21. Anwar H, Fischbacher CM, Leese G, et al. Assessment of the under-reporting of diabetes in hospital admission data: a study from the Scottish Diabetes Research Network Epidemiology Group. Diabet Med 2011;28:1514-19.

22. Shah BR, Hux JE. Quantifying the risk of infectious diseases for people with diabetes. Diabetes Care 2003;26:510-13.

23. Kornum JB, Thomsen RW, Riis A, et al. Type 2 diabetes and pneumonia outcomes: a population-based cohort study. Diabetes Care 2007;30:2251-7.

24. Benfield T, Jensen JS, Nordestgaard G. Influence of diabetes and hyperglycaemia on infectious disease hospitalization and outcomes. Diabetologia 2007;50:549-54.

25. Seshasai SR, Kaptoge S, Thompson A, et al., Emerging Risk Factors Collaboration. Diabetes mellitus, fasting glucose, and risk of cause-specific death. N Engl J Med 2011;364:829-41. 